\title{
SET MEMBERSHIP PARAMETER IDENTIFICATION WITH COMPLEX INTERVALS USING POLAR FORMS.
}

\author{
N. Ramdani, T. Raïssi, Y. Candau, A. Boudenne and L. Ibos.
}

\author{
CERTES, Université Paris XII -Val de Marne, 61 avenue du Général de Gaulle, \\ 94010 Créteil cedex, France.
}

\begin{abstract}
This paper is dedicated to bounded error identification with complex valued non-linear models. Complex intervals are characterized by using polar forms and a new inclusion function is given for the addition of sectors. The latter is expressed as an optimization problem solved analytically. The new complex interval arithmetic is used with actual data and a complex valued non-linear model for the bounded error identification of the thermal properties of materials. Copyright (C) 2005 IFAC.
\end{abstract}

Keywords : Intervals, complex variables, polar form, set membership identification, bounded noise, parameter identification, nonlinear models.

\section{INTRODUCTION}

In the literature, parameter estimation problems are usually solved by probabilistic methods where an explicit characterization of the measurement noise is assumed available. In practice, this is not usually the case for many reasons (for instance, there is a modelling error that cannot be taken into account by a random variable) and it is more natural to assume that the perturbations belong to a known set. In such a case, bounded-error approaches allow the characterization of the set of all state or parameter vectors that are compatible with the measured data, a model structure and the prior error bounds. The problem of parameter estimation in a bounded error context has been investigated by many researchers and several techniques have been established for characterizing the posterior feasible set (see e.g. Milanese, et al., 1996; and the references therein). For linear models for instance, simple-shaped forms such as ellipsoids, parallelotopes, zonotopes or boxes are used to give an enclosure of this set (Durieu, et al. 2001; Maksarov, and Norton, 2002) whereas for non-linear models, techniques based on interval analysis and constraint satisfaction problems are used (Jaulin, and Walter, 1993; Jaulin, et al. 2001; and references therein).

For many real-life engineering problems, it is more convenient to base the experimental modelling on frequency response data : the system is then described by a complex-valued model. In a bounded error context, all the uncertainties are thus described by complex sets. As a result, the derivation of an optimal inclusion function for a complex-valued nonlinear model is a major issue for ensuring success for the identification procedure. The paper is structured as follows: fundamentals about set membership identification and interval analysis are recalled in section 2. Section 3 is dedicated to complex interval analysis and contains the major contribution of this paper: polar forms are used for characterizing complex intervals and the smallest polar complex interval, called sector, containing the sum of two sectors is given. Section 4 contains the application of polar complex intervals to bounded error identification of the thermal properties of a sample material from actual data. 


\section{SET MEMBERSHIP IDENTIFICATION WITH INTERVAL ANALYSIS}

\subsection{Set membership estimation}

We denote by $\mathbf{y}_{m}(\mathbf{p})$ the model output vector, $\tilde{\mathbf{y}}$ the experimental data vector and $\mathbb{E}$ a feasible domain for output error, known prior to the identification. The feasible domain for model output is then given by

$$
\mathbb{Y}=\tilde{\mathbf{y}}+\mathbb{E}
$$

Estimating the parameter vector $\mathbf{p}$ in a bounded error context consists in determining the set $\mathbb{S}$ of all acceptable parameters

$$
\mathbb{S}=\left\{\mathbf{p} \in \mathbb{P} \mid \mathbf{y}_{m}(\mathbf{p}) \in \mathbb{Y}\right\}
$$

The characterization of the solution set $\mathbb{S}$ is a set inversion problem; a guaranteed approximation of such a set can be provided by using interval analysis.

\subsection{Interval Analysis}

Interval analysis was initially developed to take into account the quantification errors introduced by the rational representation of real numbers with computers (Moore, 1966) and was later extended to validated numerics. An interval $[a]=[\underline{a}, \bar{a}]$ is a connected and closed subset of $\mathbb{R}$. The set of all intervals of $\mathbb{R}$ is denoted by $\mathbb{I}$. Real arithmetic operations are extended to intervals. Let $\mathbf{f}: \mathbb{R}^{n} \rightarrow \mathbb{R}^{m}$, an inclusion function of $\mathbf{f}$, denoted by [f] , is defined by:

$$
\forall[\mathbf{a}] \in \mathbb{I} \mathbb{R}^{n}, \mathbf{f}([\mathbf{a}]) \subseteq[\mathbf{f}]([\mathbf{a}])
$$

An inclusion function of $\mathbf{f}$ can be obtained by replacing each occurrence of a point variable by its corresponding interval variable and by replacing each standard function by an interval evaluation. Such a function is called the natural form. In practice the inclusion function is not unique, it depends on how $\mathbf{f}$ is written.

\subsection{SIVIA}

SIVIA (Set Inversion Via Interval Analysis, (Jaulin, et al., 2001)), based on interval analysis, allows the characterization of the solution set $\mathbb{S}$, defined by (2), by computing two sets $\underline{\mathbb{S}}$ and $\overline{\mathbb{S}}$ such as:

$$
\underline{\mathbb{S}} \subseteq \mathbb{S} \subseteq \overline{\mathbb{S}}
$$

The set $\mathbb{S}$ contains all the boxes proved to be feasible. A box [p] is called:

- $\quad$ feasible, if $\left[\mathbf{y}_{m}([\mathbf{p}])\right] \subseteq \mathbb{Y}$

- $\quad$ unfeasible, if $\left[\mathbf{y}_{m}([\mathbf{p}])\right] \cap \mathbb{Y}=\varnothing$

- $\quad$ undetermined, otherwise.

The recursive algorithm SIVIA partitions the prior space $\mathbb{P}$ into boxes [p] to be submitted to these tests. Any undetermined box is bisected and tested again, unless its size is less than a precision parameter $\eta$ to be tuned by the user, which ensures that the algorithm terminates after a finite number of iterations. The outer approximation is then computed as

$$
\overline{\mathbb{S}}=\underline{\mathbb{S}} \cap \Delta \mathbb{S}
$$

where $\Delta \mathbb{S}$ is the union of all remaining undetermined boxes. Since SIVIA is a branch-andbound algorithm, its complexity is exponential with the number of parameters to be estimated, which means that it is efficient only when the dimension of $\mathbf{p}$ is low. To reduce the number of bisections in SIVIA, contractors founded on constraint propagation techniques for instance (Jaulin, et al., 2001) are used. These techniques make it possible to reduce the search box without making bisections.

\section{COMPLEX INTERVALS ANALYSIS}

The simplest complex interval approximation is the rectangular representation where a complicated shaped set is approximated by a rectangle; but the circular form, where a set is approximated by a disc, is more often used. Unfortunately, both of complex interval representations cited above are not closed with respect to the arithmetic operations $\{+,-, *, /\}$. This is due to the fact that a multiplication of a set by a complex number is a rotation, thus the result of such an operation must be wrapped in a rectangle, which introduces large pessimism. The arithmetic operation $\{*\}$ is then non minimal and a pessimism is introduced when a multiplication of two complex intervals, represented as rectangles or discs, is performed (Henrici, 1971; Rokne, and Lacaster, 1971; Nickel, 1980; Klatte, and Ullrich, 1980; Alefeld, and Herzberger, 1983; Kearfott, 1996; Petkovic, and Petkovic, 1998).

In this paper, we endeavour to extend the polar representation of complex numbers to the case of intervals. Indeed, the polar representation can be preferred for non-linear complex valued models. We will prove that both the multiplication and the division are exact operations; the result of the multiplication of two polar complex intervals is a polar interval. Nevertheless, this property is not satisfied for addition and subtraction. Consequently, 
the main contribution of this paper is the derivation of an algorithm which allows to compute the minimal polar complex interval containing the sum of two polar intervals.

\subsection{Polar representation: Definition}

Consider the intervals $[\rho] \subseteq \mathbb{R}^{+}$and $[\theta] \subseteq \mathbb{R}$, the set defined by

$$
Z=\left\{z \in \mathbb{C} \mid z=\rho e^{i \theta}, \rho \in[\rho], \theta \in[\theta]\right\}
$$

is called a polar complex interval (or a sector) denoted by $\{[\rho] ;[\theta]\}$. A polar interval can be uniquely characterized by two real intervals: its magnitude $[\rho]=\left[\rho^{-}, \rho^{+}\right], \quad$ and its angle $[\theta]=\left[\theta^{-}, \theta^{+}\right]$; as illustrated in fig. 1 , to ensure uniqueness of the representation, we can always choose the bounds of the latter interval such that

$0 \leq \theta^{+}-\theta^{-} \leq 2 \pi, \quad 0 \leq \theta^{-}<2 \pi, \quad 0 \leq \theta^{+}<4 \pi$

The set of all polar complex intervals is denoted by $\mathbb{S}(\mathbb{C})$.

\subsection{Arithmetic operations}

Let $Z_{1}=\left\{\left[\rho_{1}\right] ;\left[\theta_{1}\right]\right\}$ and $Z_{2}=\left\{\left[\rho_{2}\right] ;\left[\theta_{2}\right]\right\}$ be two sectors, the multiplication operation between $Z_{1}$ and $Z_{2}$ is defined as follows:

$$
\begin{aligned}
Z_{1} \cdot Z_{2} & \triangleq\left\{z_{1} * z_{2} \mid z_{1} \in Z_{1}, z_{2} \in Z_{2}\right\} \\
& =\left\{\begin{array}{l}
\left(\rho_{1} * \rho_{2}\right) e^{i\left(\theta_{1}+\theta_{1}\right)} \mid \\
\left(\rho_{1}, \rho_{2}, \theta_{1}, \theta_{2}\right) \in\left[\rho_{1}\right] \times\left[\rho_{2}\right] \times\left[\theta_{1}\right] \times\left[\theta_{2}\right]
\end{array}\right\} \\
& =\left\{\left[\rho_{1}\right] *\left[\rho_{2}\right] ;\left[\theta_{1}\right]+\left[\theta_{2}\right]\right\}=\{[\rho] ;[\theta]\}
\end{aligned}
$$

Since the set of the real intervals is closed with respect to addition and multiplication, the product of two sectors is also a sector; this operation is then minimal. Similarly, the division is defined by

$$
\begin{aligned}
Z_{1} / Z_{2} & =\left\{\begin{array}{l}
\left\{\rho_{1} ; \theta_{1}\right\} /\left\{\rho_{2} ; \theta_{2}\right\} \mid \\
\left\{\rho_{1} ; \theta_{1}\right\} \in Z_{1},\left\{\rho_{2} ; \theta_{2}\right\} \in Z_{2}
\end{array}\right\} \\
& =\left\{\left[\rho_{1}\right] /\left[\rho_{2}\right] ;\left[\theta_{1}\right]-\left[\theta_{2}\right]\right\}=\{[\rho] ;[\theta]\}
\end{aligned}
$$

where $0 \notin\left[\rho_{2}\right]$. We should note that the argument bounds of the result may not verify (7). In such a case, we add $2 k \pi, k \in \mathbb{Z}$ to the argument of the computed sector until (7) is met.

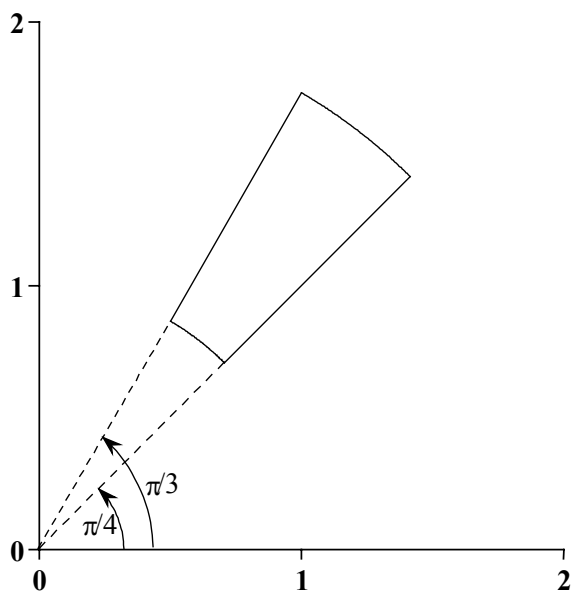

Fig. 1. A sector orientation

By contrast, the set

$$
Z_{1} \oplus Z_{2}=\left\{z_{1}+z_{2} \mid z_{1} \in Z_{1}, z_{2} \in Z_{2}\right\}
$$

known as the Minkowski sum (Farrouki, and Pottmann, 2002), is not a sector but has a complex shape; to define addition as an operation in $\mathbb{S}(\mathbb{C})$, one has to determine some element of $\mathbb{S}(\mathbb{C})$ which contains this set. Some degree of pessimism will thus be introduced. To minimize pessimism, we define $Z_{1}+Z_{2}$ as the smallest sector, in the sense of inclusion, containing $Z_{1} \oplus Z_{2}$ :

$$
Z_{1}+Z_{2}=\cap Z, \quad Z \in \mathbb{S}(\mathbb{C}), \quad Z_{1} \oplus Z_{2}=\cap Z
$$

$Z_{1}+Z_{2}$ defined in this way exists as an element of $\mathbb{S}(\mathbb{C})$, because the intersection of any number of closed boxes is a closed box in $\mathbb{R}^{2}$. Subtraction is defined in the same way, and does not in fact necessitate a separate treatment from addition, because $Z_{1}-Z_{2}=Z_{1}+\left(-Z_{2}\right)$, where $\left(-Z_{2}\right)=\left\{\mathrm{z} \mid-\mathrm{z} \in Z_{2}\right\}$; therefore we shall not mention subtraction in this paper.

\subsection{Characterization of the addition of two sectors}

Let $Z_{1}=\left\{\left[\rho_{1}\right] ;\left[\theta_{1}\right]\right\}$ and $Z_{2}=\left\{\left[\rho_{2}\right] ;\left[\theta_{2}\right]\right\}$ be two sectors and $Z$ their sum; then $Z$ can be uniquely written as $Z=\{[\rho] ;[\phi]\}$. Then the bounds $\rho$ and $\rho^{+}$of $[\rho]$ must verify

$$
\left\{\begin{array}{l}
\rho^{-}=\min _{z \in Z_{1} \oplus Z_{2}}|z| \\
\rho^{+}=\max _{z \in Z_{1} \oplus Z_{2}}|z|
\end{array}\right.
$$

with

$$
\left|\rho_{1} e^{i \theta_{1}}+\rho_{2} e^{i \theta_{2}}\right|=\sqrt{\rho_{1}^{2}+\rho_{2}^{2}+2 \rho_{1} \rho_{2} \cos \left(\theta_{1}-\theta_{2}\right)}
$$


and the function square root is monotonously increasing, solving the first of problems (12) is equivalent to solving

$$
\min _{\Omega} f\left(\rho_{1}, \rho_{2}, \theta\right)
$$

with the following definitions:

$$
\begin{gathered}
f:\left(\rho_{1}, \rho_{2}, \theta\right) \mapsto \rho_{1}^{2}+\rho_{2}^{2}+2 \rho_{1} \rho_{2} \cos (\theta) \\
\Omega=\left[\rho_{1}\right] \times\left[\rho_{2}\right] \times[\theta] \subset \mathbb{R}^{+} \times \mathbb{R}^{+} \times[0,2 \pi]
\end{gathered}
$$

with $\theta=\theta_{1}-\theta_{2}$. Thus the problem of finding $\rho^{-}$is identical to minimizing a function on a box of $\mathbb{R}^{3}$. The same applies to $\rho^{+}$, by replacing min by max. In the same way, the bounds of $[\phi]$ are solution of

$$
\left\{\begin{array}{l}
\phi^{-}=\min _{z \in Z_{1} \oplus Z_{2}} A(z) \\
\phi^{+}=\max _{z \in Z_{1} \oplus Z_{2}} A(z)
\end{array}\right.
$$

where $A(z)$, the angle of a complex $z$, is defined on $Z_{1}+Z_{2}$ such that $A(z) \in\left[\phi^{-}, \phi^{+}\right]$, this is always possible because $Z_{1} \oplus Z_{2} \subset Z$ and $Z \in \mathbb{S}(\mathbb{C})$. Denote $\quad z_{1}=\rho_{1} e^{i \theta_{1}}, \quad z_{2}=\rho_{2} e^{i \theta_{2}}$, $z=\rho e^{i \theta}=\rho_{1} e^{i \theta_{1}}+\rho_{2} e^{i \theta_{2}}$ and $x=\rho_{1} / \rho_{2}$, then

$$
\tan (\phi)=g\left(x, \theta_{1}, \theta_{2}\right)
$$

where function $g$ is defined by

$$
g\left(\rho_{1}, \rho_{2}, \theta_{1}, \theta_{2}\right)=\frac{x \sin \theta_{1}+\sin \theta_{2}}{x \cos \theta_{1}+\cos \theta_{2}}
$$

Since the derivative of function tan is strictly positive, the extrema of $A$ are also extrema of $g$. In conclusion, computing the lower and upper bounds of $[\rho]$ and $[\phi]$ are optimization problems that will be solved analytically, since they are not very difficult and the number of variables is only 3 .

\subsection{Optimality conditions}

Let $\Omega=\left[u_{1}\right] \times\left[u_{2}\right] \times\left[u_{3}\right] \subset \mathbb{R}^{3}$ and $f$ real function on $\Omega$, and consider the problem

$$
\max _{\Omega} f
$$

$\Omega$ is a compact convex set and problem (19) has a solution $\mathbf{u}^{*}=\left(u_{1}^{*}, u_{2}^{*}, u_{3}^{*}\right)$. For any index $i$, the $i^{\text {th }}$ component $u_{i}^{*}$ of $u^{*}$ must verify one of the following conditions :

$$
\begin{gathered}
\frac{\partial h}{\partial u_{i}}\left(\mathbf{u}^{*}\right)=0 \quad \text { and } \quad \frac{\partial^{2} h}{\partial u_{i}^{2}}\left(\mathbf{u}^{*}\right) \leq 0 \\
u_{i}^{*}=u_{i}^{-} \quad \text { and } \quad \frac{\partial h}{\partial u_{i}}\left(\mathbf{u}^{*}\right)<0 \\
u_{i}^{*}=u_{i}^{+} \quad \text { and } \quad \frac{\partial h}{\partial u_{i}}\left(\mathbf{u}^{*}\right)>0
\end{gathered}
$$

in the case of a minimization problem, the same conditions apply with all inequalities reversed. Observe that each of these conditions is composed of a first part which is an equation (first-order condition) and a second part which is an inequality (second-order condition). A set of $n$ first-order conditions, one for each index $i$, is an equation set, which will usually have one solution. A point of $\mathbb{R}^{n}$ which verifies, for each of its component, one of the first-order conditions will be termed a candidate. If the corresponding second-order condition is also met, it will be termed an acceptable candidate (in fact, a local optimum). The strategy used by the authors to solve (15) is to determine analytically all candidates, by examining all possible combinations of first-order conditions, then, by investigation of second-order conditions, to eliminate the candidates that can never be acceptable. The authors set up a reasonably efficient algorithm to check the acceptability of remaining candidates, and to select the optimum, by simple comparison among acceptable candidates (Candau, et al., 2005).

\subsection{Algorithms}

As an illustration of the methodology, let us consider the determination of the maximum of the magnitude of addition of two sectors. The analysis of the derivatives of (15) leads to the following candidates

$$
\begin{gathered}
\rho_{1}^{*}=\rho_{1}^{+}, \text {if } \rho_{1}^{+}+\rho_{2}^{*} \cos \theta^{*}>0 \\
\rho_{1}^{*}=\rho_{1}^{-}, \text {if } \rho_{1}^{-}+\rho_{2}^{*} \cos \theta^{*}<0 \\
\rho_{2}^{*}=\rho_{2}^{+}, \text {if } \rho_{2}^{+}+\rho_{1}^{*} \cos \theta^{*}>0 \\
\rho_{2}^{*}=\rho_{2}^{-}, \text {if } \rho_{2}^{-}+\rho_{1}^{*} \cos \theta^{*}<0 \\
\sin \theta^{*}=0, \quad \text { if } \cos \theta^{*}>0 \\
\theta^{*}=\theta^{+}, \text {if } \sin \theta^{+}<0 \\
\theta^{*}=\theta^{-}, \text {if } \sin \theta^{-}>0
\end{gathered}
$$

Each candidate is assigned a label of three numbers $i j k$, where $i=1,2, j=1,2, k=1,2,3$. For instance the candidate $(123)$ is the point $\left(\rho_{1}^{+}, \rho_{2}^{-}, \theta^{-}\right)$, its conditions of acceptability are

$$
\begin{gathered}
\rho_{1}^{+}+\rho_{2}^{-} \cos \theta^{-}>0 \\
\rho_{2}^{-}+\rho_{1}^{+} \cos \theta^{-}<0 \\
\sin \theta^{-}>0
\end{gathered}
$$


and the corresponding value for the maximum of $\rho$ is

$$
\rho(123)=\sqrt{\left(\rho_{1}^{+}\right)^{2}+\left(\rho_{2}^{-}\right)^{2}+2 \rho_{1}^{+} \rho_{2}^{-} \cos \left(\theta^{-}\right)}
$$

There are 12 candidates and 3 conditions that must be checked in order to determine the acceptability of each candidate. In fact, some conditions will never be true, or some combinations will never hold. The actual number of expressions to evaluate is in fact fairly small. Finally, four algorithms are set up for computing the extrema of both magnitude and angle of the smallest sector containing the sum of two complex sectors (Candau, et al., 2005). In the next section, this new inclusion function is used for performing set inversion via interval analysis.

\section{APPLICATION}

\subsection{Experimental procedure}

The experimental procedure under analysis hereafter is devoted to the measurement of the thermal properties of materials: the thermal diffusivity, denoted $a$, and the thermal conductivity $\lambda$, of a Polyvinylidene Fluoride (PVDF) sample are measured simultaneously by using a so-called periodic method, using multi-harmonic heating signals (Boudenne, et al., 2004). The experimental set-up is shown on figure 2 .

The PVDF sample under study is held in between a metallic rack, with a thermal grease ensuring good thermal exchange between the sample and the metallic rack. The front side of the rack, made of brass, is also fixed to a heating device (thermoelectric cooler). The rear side, made of copper, is in contact with air at ambient temperature and high vacuum. Experimental data is given as the following frequency response

$$
H_{S}(j \omega)=\frac{T_{\text {rear }}(j \omega)}{T_{\text {front }}(j \omega)}
$$

where the temperature spectra are given by the Fourier transform of the time-history signals.

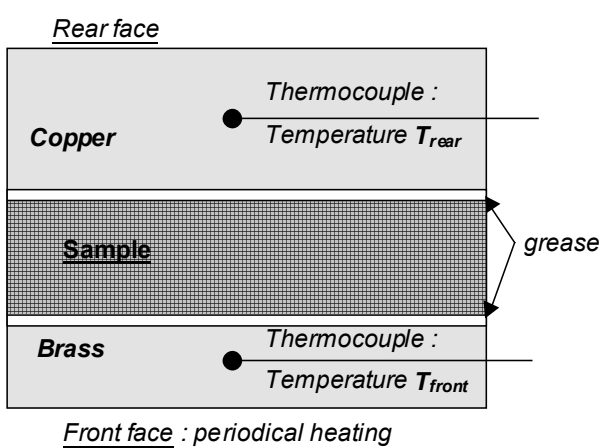

Fig. 2. The experimental set-up.
A guaranteed characterization of an earlier version of the experimental set up has already been performed in a bounded-error with set inversion and projection algorithms in (Braems, et al., 2003), but, complex intervals were approximated by rectangles. In the sequel, the authors aim to use the new inclusion functions with polar complex intervals. The latter should minimize the pessimism in interval computations.

The system under study is modelled with onedimensional quadrupoles (two-port transfer functions). The quadrupole method is well known and extensively used in thermal sciences (Wang, et $a l ., 2002)$. A "quadrupole" $\mathbf{Z}(s)$ is defined by

$$
\mathbf{Z}(s)=\left[\begin{array}{cc}
\cosh (\sqrt{\tau s}) & \frac{R}{\sqrt{\tau s}} \sinh (\sqrt{\tau s}) \\
\frac{\sqrt{\tau s}}{R} \sinh (\sqrt{\tau s}) & \cosh (\sqrt{\tau s})
\end{array}\right]
$$

where $\tau=\delta^{2} / a, \quad R=\delta / \lambda$ and $\delta$ is material thickness. For the particular case of the grease layer, which is assumed with no inertia, the relationship uses the resistance only and becomes

$$
\mathbf{Z}(s)=\left[\begin{array}{cc}
1 & R \\
0 & 1
\end{array}\right]
$$

The model transfer function is then given by

$$
H(s, \mathbf{p})=\frac{T_{\text {rear }}(s)}{T_{\text {front }}(s)}
$$

where the front temperature is given by (the $s$ symbol being removed, for convenience)

$$
\left[\begin{array}{l}
T_{\text {front }} \\
\varphi_{\text {front }}
\end{array}\right]=\mathbf{Z}_{\text {Brass }} \mathbf{Z}_{\text {Gre }} \mathbf{Z}_{\text {Sample }} \mathbf{Z}_{\text {Gre }} \mathbf{Z}_{\text {Copp }}\left[\begin{array}{c}
T_{0} \\
h T_{0}
\end{array}\right]
$$

and the rear temperature is given by

$$
\left[\begin{array}{c}
T_{\text {rear }} \\
\varphi_{\text {rear }}
\end{array}\right]=\mathbf{Z}_{\text {Copp_half }_{-}}\left[\begin{array}{c}
T_{0} \\
h T_{0}
\end{array}\right]
$$

where $h$ is a constant coefficient modelling surface heat exchanges with ambiant air, and where $T_{0}$ is rear face surface temperature.

\subsection{Bounded-error identification}

Error bounds on the experimental transfer function are calculated prior to the identification, from measurements repeated 20 times. For the sample under study, prior search space for the parameters is: 


$$
\sqrt{\tau_{p}} \in[1,30] \text { and } R_{p} \in\left[10^{-4}, 5\right]
$$

The prior search space bounds are obtained from extreme values of thermal conductivity and diffusivity found in the literature for polymeric materials, and also from the uncertainty on the material sample thickness. SIVIA with a contractor, $\eta=0.001$ and the polar inclusion functions as defined in section 3 , derives in $20 s$ the inner and outer approximations plotted in figure 3 .

The projection of the outer approximation $\overline{\mathbb{S}}$ onto the parameter axes provides an outer approximation of the uncertainty associated with each of the identified parameters

$$
\left\{\begin{array}{rlrl}
\sqrt{\tau_{p}} & \in[6.8331,7.2404] & & s^{1 / 2} \\
R_{p} & \in[0.010766,0.011409] & m^{2} K W^{-1}
\end{array}\right.
$$

thus

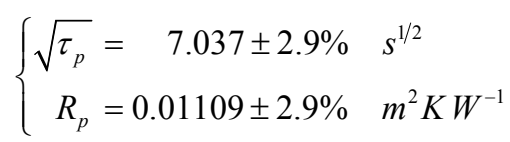

The identified values are in agreement with the values found in the literature for the material under analysis.

\section{CONCLUSION}

This paper addresses bounded error parameter identification for complex-valued non-linear models. Complex intervals are characterized with polar forms and a new inclusion function is defined for computing the smallest sector containing the sum of two polar complex intervals. The new inclusion function is used within a set inversion algorithm for the bounded error identification of thermal properties from actual data. The evaluation of the new inclusion function will be continued with new algorithms and experimental data.

\section{REFERENCES}

Boudenne, A., L. Ibos, E. Géhin and Y. Candau (2004). A simultaneous characterization of thermal conductivity and diffusivity of polymer materials by a periodic method. J. Phys. D: Appl. Phys., 37, 132-139.

Braems I., L. Jaulin, M. Keiffer, N. Ramdani and E. Walter (2003). Reliable parameter Estimation in Presence of Uncertainty. Proc. SYSID2003, 1856-1861.

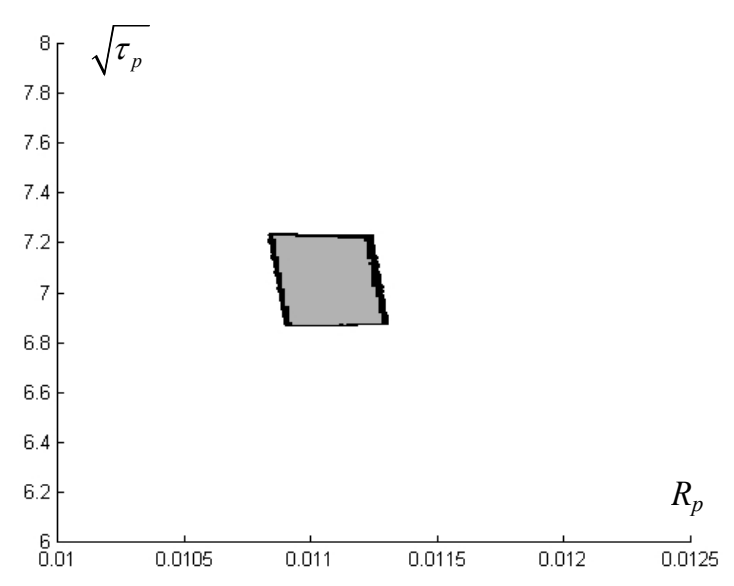

Fig. 3: Inner and outer approximations of the feasible set

Candau, Y., T. Raïssi, N. Ramdani and L. Ibos (2005). Complex interval arithmetic using polar form, Reliable Computing, submitted.

Durieu, C., E. Walter and B Polyak (2001). MultiInput Multi-Output Ellipsoidal State Bounding. J. Opt. Theory Applications, 111(2), 273-303.

Farouki, R. T. and H. Pottmann (2002). Exact Minkowski products of $\mathrm{N}$ complex disks, Reliable Computing, 8, 43-66.

Henrici, P. (1971). Circular arithmetic and the determination of polynomial zeros. Springer Lecture Notes, 228, 86-92.

Jaulin, L. and E. Walter (1993). Set inversion via interval analysis for non linear bounded-error estimation. Automatica, 29(4), 1053-1064.

Jaulin, L., M. Kieffer, O. Didrit and E. Walter (2001). Applied Interval Analysis. London: Springer.

Klatte, R. and C. Ullrich (1980). Complex sector arithmetic. Computing, 24(2-3), 139-148.

Maksarov, D.G. and J.P. Norton (2002). Computationally efficient algorithms for state estimation with ellipsoidal approximation. Int. J. Adapt. Control Signal Process, 16(6), 411-434.

Milanese, M., J.P. Norton, H. Piet-Lahanier and E. Walter (1996). Bounding approaches to system identification. New York: Plenum.

Moore, R. E. (1966). Interval analysis. Englewood Cliffs, NJ, Prentice-Hall.

Nickel, K. (1980). Arithmetic of complex sets, Computing, 24, 97-105.

Petkovic, M. S. and L. D. Petkovic (1998). Complex interval arithmetic and its applications, Wiley$\mathrm{VCH}$, Berlin.

Rokne, J. and P. Lancaster (1971). Complex interval arithmetic. ACM 14, 111-112.

Wang, H., A. Degiovanni and C. Moyne (2002). Periodic thermal contact: a quadrupole model and an experiment, Int. J. Therm. Sci., 41, 125135. 